\title{
Neuro-based Olfactory Model for Artificial Organoleptic Tests
}

\author{
$\mathrm{Zu} \mathrm{Soh}{ }^{\dagger}$, Toshio Tsuji ${ }^{\dagger}$, Noboru Takiguchi ${ }^{\dagger \dagger}$, and Hisao Ohtake ${ }^{\dagger \dagger}$ \\ $†$ Graduate School of Engineering, Hiroshima Univ., Hiroshima 739-8527, Japan \\ $\dagger \dagger$ Graduate School of Advanced Sciences of Matter, Hiroshima Univ., Hiroshima 739-8530, Japan \\ †† Graduate School of Engineering, Osaka Univ., Osaka 565-0871, Japan
}

\begin{abstract}
Recently, the demand for odor processing apparatus in the fragrance and food industries has increased. In this paper, we construct a neural network model of the olfactory system as basis for artificial organoleptic tests that combines possesses the advantages of both human sensory evaluation and machine olfaction. The simulation results indicate that the model can predict odor coding on the glomeruli by appropriately adjusting the parameters involved. Further, the model can simulate the feature extraction ability known Attention.
\end{abstract}

\section{Introduction}

As considerable evidence has been presented to show that odors have an effect on memory and emotions [1], the importance of odors has begun to be recognized beyond their role as components of flavor. For this reason, the demand for odor processing apparatus in the fragrance and food industries is increasing $[2]$.

Two methods of odor assessment developed so far are the sensory evaluation method and machine olfaction [2]. Generally, the sensory evaluation method is employed because it is based on the characteristics of human perception, although, individual differences due to factors such as personal preference or physical condition can affect the evaluation results. Machine olfaction, in contrast, is an objective assessment method; nevertheless, it tends to ignore the nature of odor perception. Accordingly, a novel odor assessment method combining the advantages of both human sensory evaluation and machine olfaction making it suitable for artificial organoleptic tests is required. To develop such a method, it is first necessary to predict how odorant information is coded in the brain to obtain the perception characteristics of animals. The mechanisms of feature extraction from the neuro-coded odor information must then also be predicted.

Although the ideal scenario would be to analyze the human olfactory system, current biological knowledge regarding the coding manner of odorant information in the human brain remains limited. Given this restriction, the present study focuses on the olfactory system of mice.

An odor is a combination of more than 400,000 kinds of odorant molecules. Mice have approximately 1,000 kinds of odorant receptors, each of which is responsible for detecting a specific group of odorant molecules [3]. The outputs of the receptor neurons evoke an odor-specific activity pattern on glomeruli $[4,5]$. As this activity pattern represents fundamental information for odor recognition, it is considered closely linked to the characteristics of perception. We constructed a neural network model [6] using biological data on glomerular activity patterns [5] as inputs, and attempted to simulate the perception characteristics of mice. The model also employed a feature extraction mechanism for Attention [7] in which the mice would focus on some of the important molecules in odors. The results of the computer simulation were compared with ones of behavioral experiments [7], and it was confirmed that the model could predict the discrimination ability of mice. However, the scope of our model was limited to odorants with known glomerular activity patterns.

In this paper, we report on the extension of the model to enable the prediction of glomerular activity patterns from odorant properties. For this purpose, 3-layered feed-forward neural network was added and trained by using a known biological data set. Predicted activity patterns were ued as the input of the model for further Attention processing. The simulation results were then examined through comparison with the odor discrimination rates obtained from behavioral experiments on mice. 


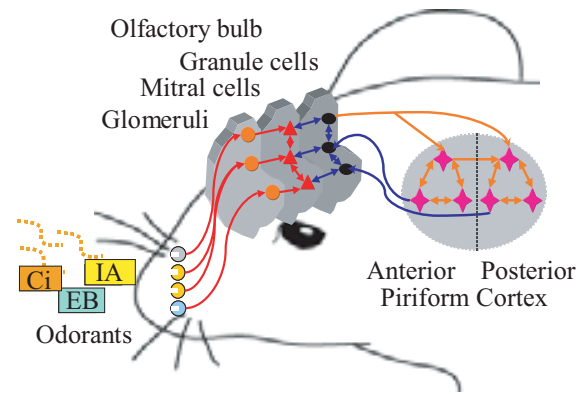

Figure 1: The olfactory system of mice.

\section{Biological insight}

\subsection{Olfactory system of mice}

Fig. 1 shows the basic structure of the olfactory system in mice, which consists of three parts: receptor neurons, the olfactory bulb and the piriform cortex. Receptor neurons that distributed on the surface of the nasal chamber express single receptor from among 1,000 different ones, and bind to specific odorants [3]. When odorant molecules bind to the receptor, its neuron is activated and sends signals to the olfactory bulb. The axons from the receptors that express the same gene terminate at the same point on the surface of the olfactory bulb [4]. The terminals of these axons form a small, round cluster called a glomerulus. A 2D map of glomerular distribution can be associated with receptor genes as well as odorants, and is thus called an odor map [4].

Besides the glomeruli, mitral cells and granule cells are the principal neurons in the olfactory bulb. Signals from the glomeruli are inputted to the mitral cells, which are interconnected via the excitatory synapse. The granule cells receive inputs from the mitral cells and send inhibitory signals back to them. In general, the olfactory bulb is considered to perform feature extraction [8].

The mitral cells transmit the signal to the pyramidal cells in the piriform cortex, which then transmit signals back to the granule cells in the olfactory bulb and indirectly inhibit the mitral cells. The piriform cortex is divided into the anterior piriform cortex (APC) and the posterior piriform cortex (PPC); the division of their functions remains slightly unclear. Generally, the piriform cortex is believed to be responsible for the identification of odors [9].

\subsection{Attention mechanism in the olfactory system}

Okuhara et al. [7] conducted a series of odor discrimination experiments on mice [7]. First, the mice

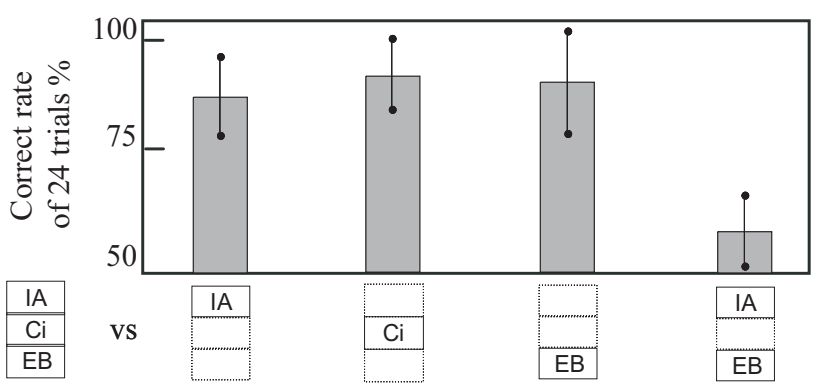

Figure 2: Results of the odor discrimination experiment.

were trained to select a rewarded odor, such as [IA, Ci, EB] composed of three types of odorant. They were then required to discriminate among other odors that contained elements in odor [IA, Ci, EB], such as [IA] or [IA, EB]. Fig. 2 outlines the results of the odor discrimination experiment performed with 10 mice, and indicates that most of them had difficulty in discriminating between [IA, EB] and [IA, Ci, EB]. This implies that they focused on a combination of the odorants [IA] and [EB] when learning the odor [IA, Ci, EB]. This mechanism is called Attention, and contributes significantly to the odor perception characteristics of mice as described above.

\section{Model of the olfactory system of mice}

\subsection{Structure of the proposed model}

Fig. 3 shows the structure of the proposed neural network model, which consists of three parts: odor reception, the olfactory bulb and the piriform cortex.

The odor reception model is a feed-forward neural network of 3 layers: which are the preprocessing layer $(l=1)$, the odorant layer $(l=2)$ and the receptor layer $(l=3)$. The neuron populations for each layer $\left({ }^{l} N\right)$ are ${ }^{1} N=80,{ }^{2} N=500$, and ${ }^{3} N=1,805$, respectively. The olfactory bulb model consists of the Glomerular layer $(l=4)$, the Mitral layer $(l=5)$, and the Granule layer $(l=6)$. The neuron populations in the olfactory bulb are ${ }^{4} N={ }^{5} N={ }^{6} N=$ 1,805 , These populations were determined based on the actual number of glomeruli distributed on the olfactory bulb [10].

The piriform cortex model consists of an $A P C$ layer $(l=7)$ and a $P P C$ layer $(l=8)$ corresponding to the anterior piriform cortex and the posterior piriform cortex, respectively. The neuron populations of the $A P C$ and the $P P C$ layers are ${ }^{7} N=1,000$ and ${ }^{8} N=$ 100 , respectively. 


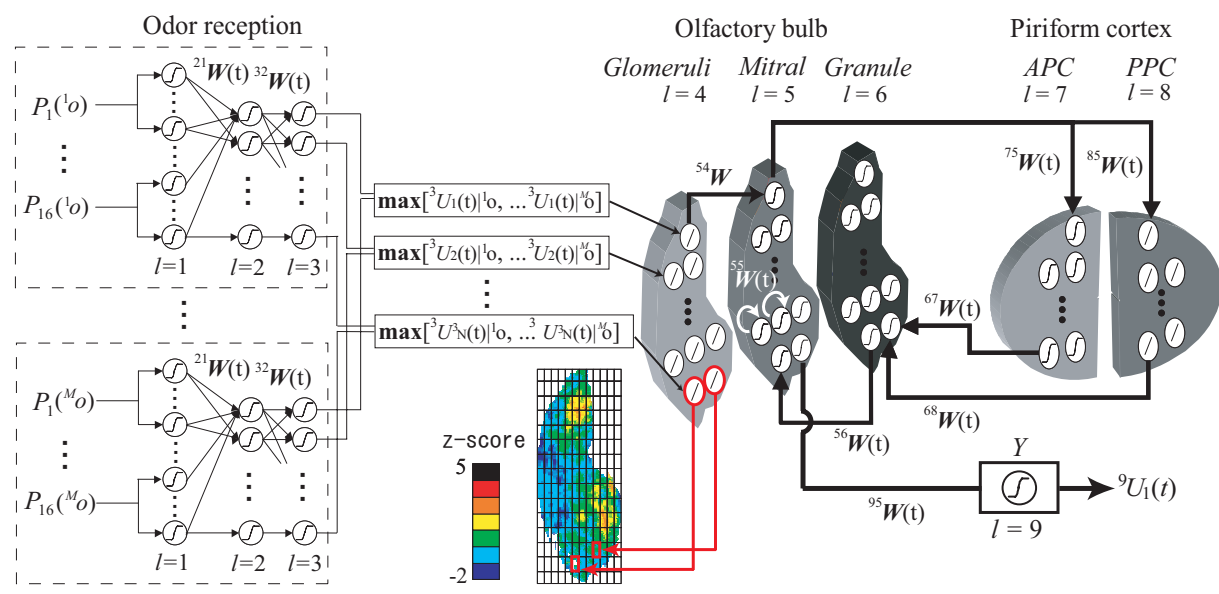

Figure 3: Structure of the proposed model. The activity pattern of glomeruli is cited from literature [5].

The connections between each layer in the olfactory bulb model and the piriform cortex model are set up based on the structure of the olfactory system described in Section 2.1, with the exception that the interconnections in each layer are not included in the model for simplification. In addition, a single neuron layer $Y(l=9)$ is artificially introduced as the output of the model.

The model takes the odorant properties as the input from the preprocessing layer. Since there are approximately 400,000 kinds of odorant (forming extremely high-dimensional information), it is impossible to input the odorant information to the model through binary coding. The properties of each odorant are therefore broken down into 16 numeric properties as listed by Johnson et al. [11] along with their corresponding activity patterns.

In order to normalize the properties with different units and orders, the preprocessing layer converts the value of the properties into activated neuron numbers. This method is introduced based on the concept of population coding [12]. The neurons in the preprocessing layer are divided into 16 groups, each of which receives a different kind of odorant property. The input to the neurons in the preprocessing layer is given by the following equation:

${ }^{1} u_{s}(t)=P_{i}\left({ }^{m} o\right), \quad(s=(i-1) K+k, k=1,2 \ldots K)$,

where ${ }^{1} u_{s}(t)$ is the input to the $s^{\text {th }}$ neuron in the preprocessing layer $(l=1)$ at time step $t,{ }^{m_{O}}$ is the $m^{\text {th }}$ odorant in odor $O, P_{i}$ is the $i^{\text {th }}$ numeric property of the odorant ${ }^{m} O$, and $\mathrm{K}$ is the maximum number of neurons responsible for property $P_{i}$. The activities of the neurons are given by the following sigmoid function:

$$
{ }^{1} U_{s}(t)=\frac{1}{1+\exp \left\{-{ }^{1} \epsilon_{s}\left({ }^{1} u_{s}(t)-{ }^{1} \theta_{s}\right)\right\}} .
$$

The outputs of sigmoid neurons in other layers are also calculated using the above equation. The threshold ${ }^{1} \theta_{s}$ and the gradient ${ }^{1} \epsilon_{s}$ of the sigmoid function are determined according to the corresponding property by the following equation:

$$
\begin{aligned}
{ }^{1} \theta_{s} & =k \frac{\left(P_{i, \max }-P_{i, \min }\right)}{K}, \\
{ }^{1} \epsilon_{s} & =C_{s} \frac{\left(P_{i, \max }-P_{i, \min }\right)}{K},
\end{aligned}
$$

where $P_{i, \max }$ and $P_{i, \text { min }}$ are the maximum and minimum values of the property $P_{i}$ in an odorant data set, and $C_{s}$ is a constant.

The output of the preprocessing layer $(l=1)$ is inputted to the odorant layer $(l=2)$ through a connective weight matrix ${ }^{1} \mathbf{W}(t)$. The input to the odorant layer is given by the following equation:

$$
{ }^{2} u_{n}(t)=\sum_{s}{ }^{21} w_{n s}(t){ }^{1} U_{s}(t)
$$

where ${ }^{2} u_{n}(t)$ is the input to the $n^{\text {th }}$ neuron in the odorant layer, and ${ }^{1} w_{n s}(t)$ is the connective weight between neuron units $n$ and $s$, which is an element in the connective weight matrix ${ }^{21} \mathbf{W}(t)$.

The output of the odorant layer is inputted to the receptor layer $(l=3)$ through ${ }^{32} \mathbf{W}(t)$ in the same manner as the equation (5).

The output of the receptor layer $(l=3)$ is passed to the glomerular layer $(l=4)$. According to Lin et al. [13], the glomerular activity of an odorant mixture $O$ can be represented by binary addition of the activities evoked by its odorant components. Thus, the input and output of the Glomerular layer is determined by the following equation:

$$
{ }^{4} u_{e}(t)=\max \left[{ }^{3} U_{r}(t)\left|{ }_{1} \ldots{ }^{3} U_{r}(t)\right|{ }_{M_{o}}\right]
$$


The output ${ }^{4} U_{e}(t)$, which is calculated by equation (2), is an element in the activity pattern vector ${ }^{4} U(t)$. Each element corresponds to a divided lattice of the activity patterns provided by Johnson et al. [5], as shown in Fig. 3.

The output of the Glomerular layer is input to the Mitral layer on a one-to-one basis. The detailed structure of the olfactory bulb and piriform cortex part is described in our previous paper [6], where the inputs and outputs of each layer are determined in the same manner as described above.

The inputs of the newly introduced $Y(l=9)$ layer represent the output of the model. Its output is determined by the input from Mitral layer as the following equation:

$$
{ }^{9} U_{1}(t)=\frac{\exp \left\{-{ }^{9} \epsilon_{1}\left({ }^{9} u_{1}(t)-{ }^{9} \theta_{1}\right)\right\}}{1+\exp \left\{-{ }^{9} \epsilon_{1}\left({ }^{9} u_{1}(t)-{ }^{9} \theta_{1}\right)\right\}} .
$$

To predict the glomerular activity patterns and perception characteristics affected by attention, the connective weights appearing in each equation must be appropriately adjusted. The next subsection describes the learning algorithm of the model.

\subsection{Algorithm of the learning phase}

The learning algorithm consists of 2 steps, whose details are described in this subsection.

\subsubsection{The $1^{\text {st }}$ step of the learning phase}

In the $1^{\text {st }}$ step, the connective weights ${ }^{21} \mathbf{W}(t)$ and ${ }^{32} \mathbf{W}(t)$ are adjusted for accurate prediction of the activity patterns for the Glomerular layer from the input odorants' properties as given by the training set. The connective weights are adjusted to minimize the error energy $E$ :

$$
E=\frac{1}{2} \sum_{i}^{4} e_{i}=\frac{1}{2} \sum_{i}^{4}\left(U_{i}^{4}-a_{i}\right)^{2},
$$

where $e_{i}$ is the mean square error (MSE); $U_{i}^{4}$ is the output of the glomerular layer, and $a_{i}$ is the activity of actual glomeruli in the $i^{\text {th }}$ lattice. For the implementation of weight adjustment, the RPROP algorithm proposed by Riedmiller et al. [14] is utilized. This algorithm allows fast error convergence with a reasonable computer memory requirement. The connective weights are iteratively adjusted until a preset maximum iteration number is reached.

\subsubsection{The $2^{\text {nd }}$ step of the learning phase}

In the $2^{\text {st }}$ step, the connective weights in the olfactory bulb and piriform cortex are modulated based on the algorithm proposed in [6]. Since most of the computational functions of the olfactory system, especially the connection from the piriform cortex to the olfactory bulb, are not yet clearly understood, signal transduction or connective weight modulation are hypothesized based on the odor discrimination experiment outlined in Section 2.2 [7]. We assume that the connection from the piriform cortex to the olfactory bulb plays a role in extracting the most activated regions in the glomeruli. With regard to these assumptions, we propose a learning algorithm that consists of 3 steps outlined below.

In the $1^{\text {st }}$ step, the connective weights ${ }^{75} \mathbf{W}(t)$ and ${ }^{67} \mathbf{W}(t)$ are modulated to subtract the background activity from the activated part of the Mitral layer using the following equation:

$$
\begin{aligned}
& { }^{75} W_{z b}(t+1)=\alpha^{75} W_{z b}(t)+\left.\left.\beta^{5} U_{b}(t)\right|_{A} O^{7} U_{z}(t)\right|_{{ }_{A}}, \\
& { }^{67} W_{g z}(t+1)=\alpha^{67} W_{g z}(t)+\left.\left.\beta^{7} U_{z}(t)\right|_{{ }_{A}} O^{5} U_{b}(t)\right|_{\text {back }},
\end{aligned}
$$

where $\alpha$ denotes the forgetting term, and $\beta$ is the learning rate. As a result, when an odor $\mathrm{A}$ is input through the connective weights ${ }^{75} W(t)$ and ${ }^{67} W(t)$, the Granule layer can inhibit the background activity of the Mitral layer.

In the $2^{\text {nd }}$ step, the most activated neurons in the Glomerular layer are extracted. The connective weights between the Mitral layer and the $P P C$ layer are assumed to form a competitive system, and the corresponding connective weights are adjusted according to Amari et al. [15].

In the $3^{\text {rd }}$ step, the activity pattern of the Mitral layer resulting from the $2^{\text {nd }}$ step is memorized by adjusting the connective weights ${ }^{95} \mathbf{W}(t)$ as in the following equation:

$$
{ }^{95} W_{1 b}(t)=\left.{ }^{5} U_{b}(t)\right|_{{ }_{A}} O .
$$

This adjustment enables the model to compare the features of the memorized odor to the inputted odors using the comparison algorithm described in the next subsection.

\subsection{Comparison algorithm}

In the comparison phase, an arbitrary odor B is inputted to the model. The input to the $Y$ layer can be calculated as follows:

$$
\begin{aligned}
\left.{ }^{9} u(t)\right|_{B O} & =\left.\sum_{b}{ }^{95} W_{1 b}(t)^{5} U_{b}(t)\right|_{B^{B}} \\
& =\left.\sum_{b}{ }^{5} U_{b}(t)||_{A}{ }^{5} U_{b}(t)\right|_{B} O .
\end{aligned}
$$




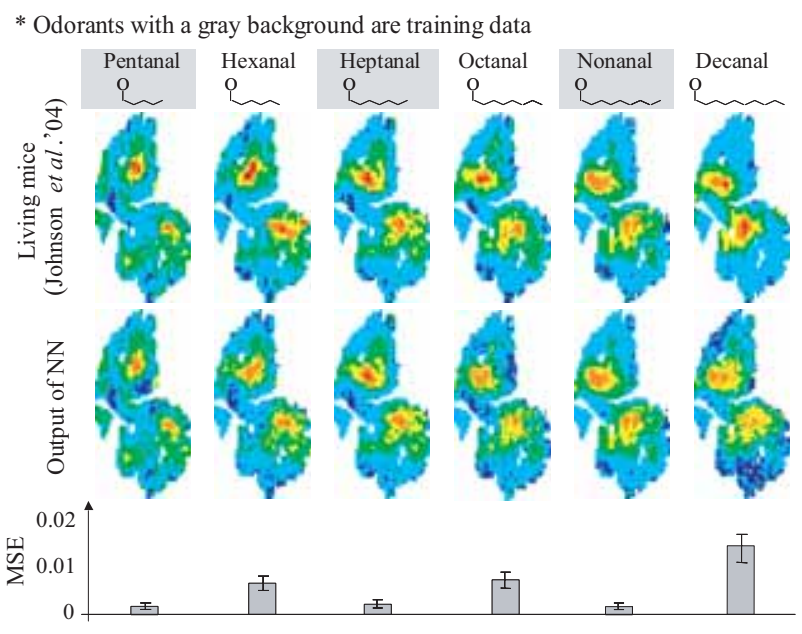

Figure 4: Simulation results of aldehydes.

Accordingly, calculating the input to the $Y$ layer is equivalent to calculating the correlation between the current output $\left.{ }^{5} U_{b}(t)\right|_{B} O$ and the memorized output $\left.{ }^{5} U_{b}(t)\right|_{A} O$ of the Mitral layer. The correlation is then converted to an index of dissimilarity by equation (7). It can be assumed that the mice tend to make wrong decisions when the outputs of the Mitral layer are similar. Accordingly, the output of the model is considered to correspond with the results of the odor discrimination experiments on mice [7].

\section{Simulation}

This section describes the simulations performed based on the algorithm described in the previous section.

\subsection{The $1^{\text {st }}$ step of the simulation}

First, data on 70 odorants were selected from the 365 odorants provided by Johnson et al. [11] as a training data set. Then, 3 odorants with identical structures but different carbon numbers were chosen and added to the set.

Fig. 4 shows the outputs of the model after the training is completed. In Fig. 4, the molecules in the uppermost row are the inputted odorants followed by the actual activity patterns [5], the output of the model, and the a graph of the mean square errors (MSEs). The odorants with a gray background are included in the training set, while those with no background color are untrained odorants. This figure indicates that the model successfully predicted the activity patterns in the training data set with an prediction error MSE of below 0.002 . The predicted activity patterns of untrained odorants were also close to the actual activity patterns with MSEs ranging from about

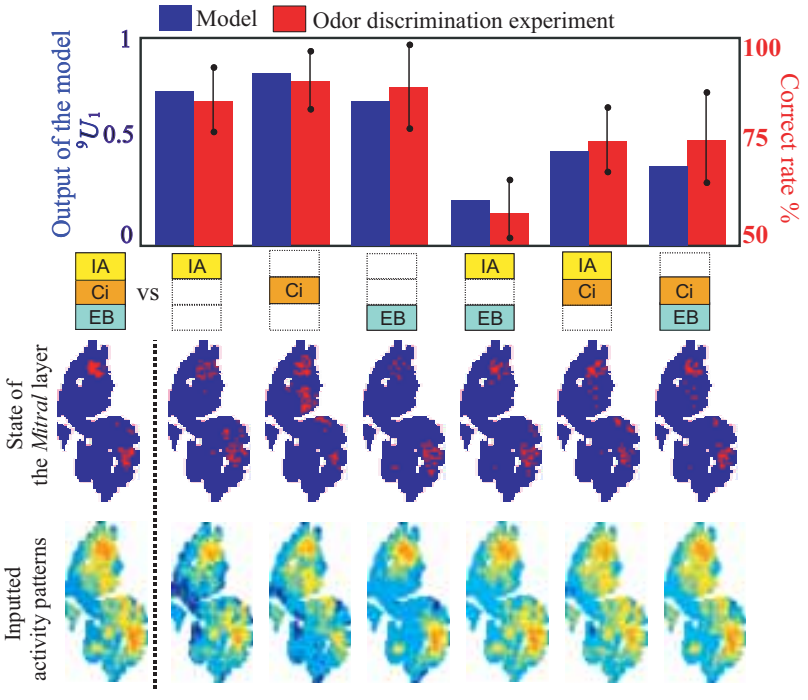

Figure 5: Comparison between the results of the behavior experiments.

0.007 to 0.015 . Consequently, the model is capable of predicting the tendency for the activated parts of glomeruli to shift continuously along with the carbon number [5].

\section{2 $\quad 2^{\text {nd }}$ step of the simulation}

In the learning phase, an odor [IA, Ci, EB], representing an odorant mixture composed of isoamyl acetate, citral, and ethyl butyrate, is input to the proposed model. The connective weights are then adjusted according to the learning algorithm described in Section 3.2.2. The initial values of the connective weights are determined by uniform random values ranging between $-10^{-5}$ and $10^{-5}$.

After the learning phase, 6 different odors [IA], [EB], [Ci], [IA, EB], [IA, Ci], and [Ci, EB] are input to the model. Then, the output of the neuron in the $Y$ layer is compared with the correct rates in odor discrimination experiments on the mice. In this step, the connective weights are fixed on the values determined in the $1^{\text {st }}$ step of the simulation.

The outputs of the Glomerular layer to each odor are shown in the bottom row in Fig. 5. The outputs of the Mitral layer after the $2^{\text {nd }}$ learning phase are shown in the middle row, and the outputs of the neurons in the $Y$ layer are plotted at the top. The discrimination rates obtained from the odor discrimination experiment on the mice are also plotted beside the output of the $Y$ layer.

Comparing the activity patterns of the Glomerular layer to those of the Mitral layer shows that the activated region becomes narrow, but its activity becomes stronger, which means that most activated regions in 
the Glomerular layer were extracted. Fig. 5 indicates a similar tendency between the correct rates and the output of the neuron in the $Y$ layer; the higher the output, the higher the correct rate. From these results, we can conclude that the model is capable of account for the perception characteristics of mice to a certain extent through the assumed Attention mechanism.

\section{Conclusion}

In this paper, we propose a neural network model of the olfactory system of mice. Utilizing this model, we tried to predict the activity pattern in glomeruli evoked by odorants. The simulation results indicated that the model was capable of predicting the activity patterns of untrained odors with different carbon numbers, and showed consistency with those of odor discrimination experiments on mice. This ability to predict perception characteristics makes the model suitable as a basis for artificial organoleptic tests.

However, odors in nature are composed of odorants in different concentrations, which is not accounted for in the proposed model. Future studies must therefore include the odor coding manner for different concentrations. In addition, since the simulations were performed only with on odorants [IA], [Ci], and [EB] using limited experimental data, further behavioral experiments and simulation need to be performed on other odorants to verify the ability of the model.

\section{Acknowledgments.}

This work was partially supported by the 21st Century COE Program of JSPS (the Japan Society for the Promotion of Science) on Hyper Human Technology toward the 21st Century Industrial Revolution.

\section{References}

[1] R.S. Herz and T. Engen, "Odor memory: review and analysis," Psychonomic Bulletin and Review, Vol. 3, No. 3, pp. 300-313, 1996.

[2] R. Gutierrez-Osuna, "Pattern Analysis for Machine Olfaction: A Review," IEEE Sensors Journal, Vol. 2, No. 3, pp. 189-202, 2002.

[3] L. Buck and R. Axel "A novel multigene family may encode odorant receptors: a molecular basis for odor recognition," Cell, Vol. 65, pp.175-187, 1991.

[4] K. Mori and Y. Yoshihara "Molecular recognition and olfactory processing in the mammalian olfactory system," Progress in Neurobiology, Vol. 45, pp. 585-619, 1995.
[5] B.A. Johnson and M. Leon "Modular glomerular representations of odorants in the rat olfactory bulb and the effects of stimulus concentration," The Journal of Comparative Neurology, Vol. 422, pp. 496-509.

[6] Z. Soh, T. Tsuji, N. Takiguchi, H. Ohtake "A neural network model of the olfactory system of mice Simulated the tendency of attention behavior," The 13th International Symposium on Artificial Life and Robotics, pp. 537-542, 2008.

[7] K. Okuhara and T. Nakamura "Explore algorithms in olfactory system of mice," Software Biology, Vol.3, pp.20-25, 2005. (in Japanese)

[8] O. Hoshino, Y. Kashimori, and T. Kambara, "Feature extraction from mixed odor stimuli based on spatio-temporalrepresentation of odors in olfactory bulb," International Conference on Neural Networks, vol. 1, pp. 344-349, 1997.

[9] D.A. Wilson, "Receptive Fields in the Rat Piriform Cortex," Chemical Senses, Vol. 26, No. 5, pp. 577 $584,2001$.

[10] B.A. Johnson, H. Farahbod, Z. Xu "Local and global chemotopic organization: general features of the glomerular representations of aliphatic odorants differing in carbon number," The Journal of Comparative Neurology, Vol. 480, No. 2, pp. 234$249,2004$.

[11] M. Leon, B. A. Johnson, "Glomerular Response Archive," http://leonserver.bio.uci.edu/.

[12] A.P. Georgopoulos, A.B. Schwartz, R.E. Kettner, "Neuronal population coding of movement direction," Science, Vol. 233, Issue. 4771, pp. 1416 1419, 1986.

[13] D.Y. Lin, S.D. Shea, L.C. Katz, "Representation of natural stimuli in the rodent main olfactory bulb," Neuron, Vol.50, No. 6, pp. 816-818, 2006.

[14] M. Riedmiller and H. Braun, "A direct adaptive method for faster backpropagation learning: The RPROP algorithm," Proceedings of the IEEE International Conference on Neural Networks San Francisco, pp. 586-591, 1993.

[15] S. Amari, M.A. Arbib, "Computation and cooperation in neural nets," Systems Neuroscience, Academic Press, pp. 67-117, 1977. 